\title{
First manifestation of citrullinemia type I as Sandifer syndrome
}

\author{
Mustafa Kılıç ${ }^{1}$ Esma Altınel-Açoğlu², Pelin Zorlu², Deniz Yüksel³, Selda Bülbül4, \\ Johannes Haeberle ${ }^{5}$ \\ ${ }^{2}$ Department of Pediatrics ${ }^{1}$ Divisions of Metabolism and ${ }^{3}$ Pediatric Neurology, Dr. Sami Ulus Children Hospital, Ankara; \\ ${ }^{4}$ Department of Pediatric Metabolism, Kırıkkale University, Faculty of Medicine, Kırıkkale, Turkey; ${ }^{5}$ Division of Metabolism \\ and Children's Research Center, University Children's Hospital, Zurich, Switzerland. \\ E-mail: kilickorkmaz@yahoo.com.tr
}

Received: 4th January 2017, Revised: 31st May 2017, Accepted: 31st May 2017

SUMMARY: Kılıç M, Altınel-Açoğlu E, Zorlu P, Yüksel D, Bülbül S, Haeberle J. First manifestation of citrullinemia type I as Sandifer syndrome. Turk J Pediatr 2017; 59: 696-698.

We report an eleven-month-old infant girl who presented as Sandifer syndrome clinically but was later diagnosed with citrullinemia type I. Metabolic evaluation and molecular analysis confirmed the correct diagnosis. Despite the fact that many patients are already known in the literature, this is the first report of a Sandifer syndrome-like presentation of citrullinemia type I. This paper suggests that suspicion of Sandifer syndrome should also lead to inclusion of urea cycle disorders in the list of differential diagnoses.

Key words: citrullinemia type I, Sandifer syndrome, hyperammonemia, vomiting.

Citrullinemia type I (CTLN1, OMIM 215700) is a rare autosomal recessive urea cycle disorder caused by the deficiency of argininosuccinate synthetase due to mutations in the ASS1 gene. Citrullinemia type I can have different presentations including acute neonatal-onset (usually referred to as classical citrullinemia) or milder late-onset, a form in which patients remain without symptoms and never experience hyperammonemia and finally a form in which women have onset of severe symptoms during pregnancy or post partum. ${ }^{1}$ Sandifer syndrome is a dystonic movement disorder in infants with gastroesophageal reflux (GER). We report an 11-month-old girl who presented with Sandifer syndrome clinically but was later diagnosed with citrullinemia type I.

\section{Case Report}

An eleven-month-old Turkish girl was brought to our hospital with complaints of vomiting in addition to abnormal head posture. She was the first child of non-consanguineous parents and there was no history of sibling deaths. Vomiting had been present for three months, and in addition, during the last three days before admission abnormal head posture developed. The perinatal period and other previous postnatal history were normal. Because of torticollis and GER, Sandifer syndrome was suspected and sodium-alginate therapy was started at another hospital. On physical examination, her head was tilted to the right, and stereotypic hand movements and mild muscular hypotonia were present (Fig. 1a). Growth parameters and eye examination were normal. Laboratory examinations including blood count, liver transaminases and kidney function tests, C-reactive protein, arterial and venous blood gases, creatine kinase, urine analyses and culture, X-ray of the cervical spine, neck and abdominal ultrasound, barium esophagography, brain MRI and electroencephalogram were all normal except for hyperammonemia (461 $\mu \mathrm{mol} / \mathrm{l}, \mathrm{N}<55)$. Quantitative plasma amino acid analyses showed elevated levels of plasma citrulline (maximum $1303 \mu \mathrm{mol} / \mathrm{L}$, $\mathrm{N}$ 3-35) and no argininosuccinic acid. Compound heterozygous c.688G $>\mathrm{A} / \mathrm{c} .793 \mathrm{C}>\mathrm{T}$ (p.Gly230Arg/p.Arg265Cys) mutations were detected in ASS1 gene confirming a diagnosis of citrullinemia type I. Thereafter, treatment with low-protein diet, sodium benzoate and arginine was initiated, which was well tolerated 


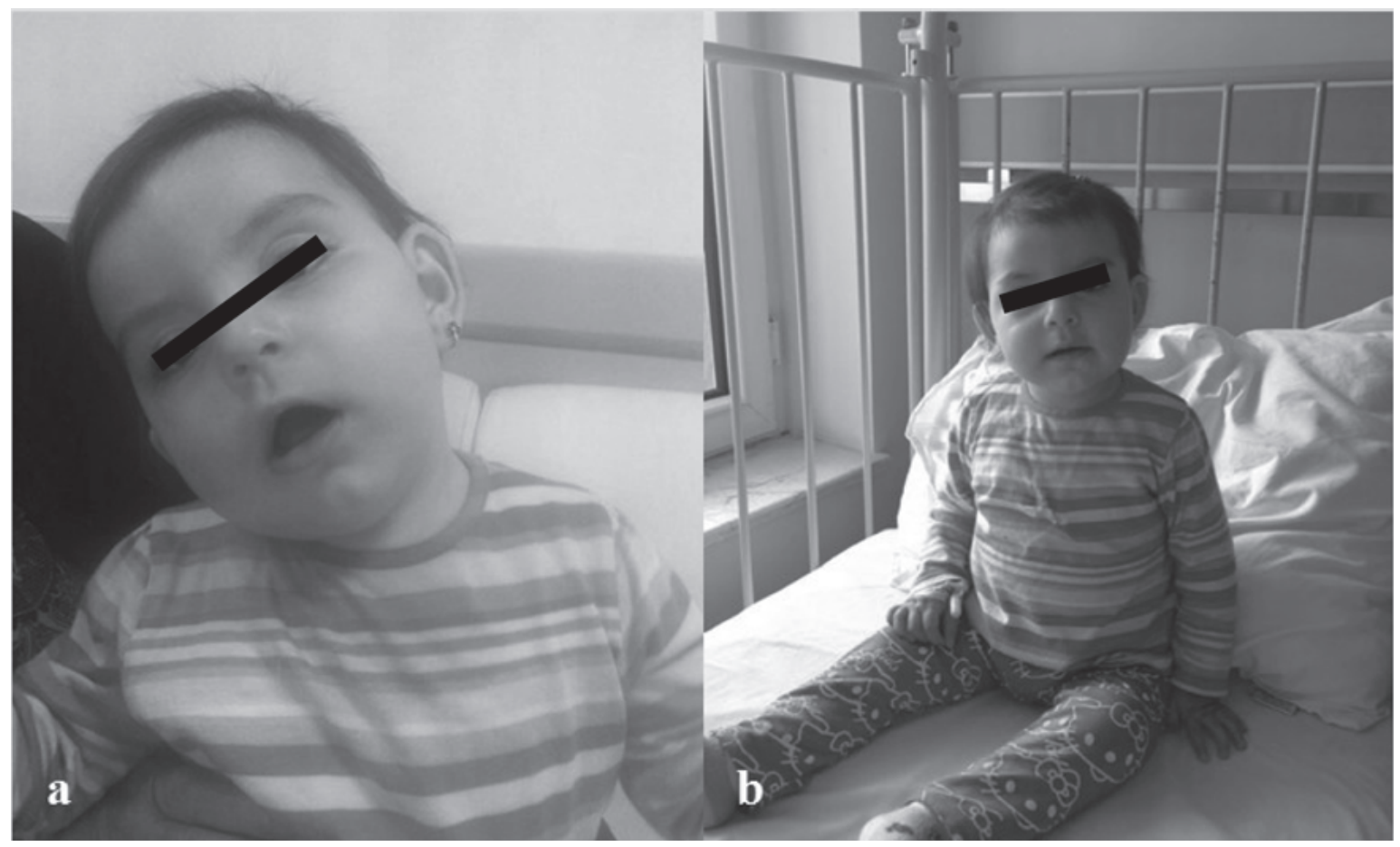

Fig. 1. Citrullinemia type I patient at 11-month-old a) before treatment, b) after treatment.

and led to a decrease of the ammonia level (97 $\mu \mathrm{mol} / \mathrm{L})$ and improvement of clinical symptoms (Fig. 1b). She received a related living-donor liver transplantation at the age of two years. The outcome was successful and she was free of diet and drugs except tacrolimus.

Written consent from the parents to publish this case including her photograph was obtained.

\section{Discussion}

Citrullinemia type I can present with a broad clinical spectrum including persistent hiccups, transient fulminant liver failure, stroke and postpartum psychosis. ${ }^{2-6}$ However, there is no report yet on a Sandifer syndromelike presentation as a manifesting sign of citrullinemia type I in the literature. Our patient presented with vomiting, abnormal head posture and was misdiagnosed with Sandifer syndrome at the first evaluation. In spite of treatment for GER, her clinical signs remained unchanged. Plasma ammonia levels finally led to the correct diagnosis of a urea cycle disorder and further metabolic and genetic evaluation confirmed presence of citrullinemia type I in this patient. After this diagnosis was made and appropriate treatment was started, symptoms of the patient disappeared. Plasma ammonia was measured due to the overall unclear situation in the patient and should be regarded as an important laboratory clue for the work-up of unexplained vomiting and/or unexplained neurological signs in infants (and beyond). Metabolic disorders should be included in the differential diagnoses of unexplained vomiting.

In Turkey, only four disorders (congenital hypothyroidism, phenylketonuria, biotinidase deficiency and cystic fibrosis) are included in the general newborn screening program. However selective screening for metabolic disorders should be done for the early diagnosis in particular to identify late-onset patients with unusual presentations, like in the present patient. Although many patients with citrullinemia type I have already been described, Sandifer syndrome-like presentation has not yet been reported. In conclusion, we suggest that urea cycle disorders should also be considered in the suspicion of Sandifer syndrome in making the differential diagnosis.

\section{Acknowledgements}

We thank the family of the patient for their participation involving informed consent and permission to publish the photograph of their daughter. 


\section{REFERENCES}

1. Quinonez SC, Thoene JG. Citrullinemia Type I. In: Pagon RA, Adam MP, Ardinger $\mathrm{HH}$, Wallace SE, Amemiya A, Bean LJH, Bird TD, Ledbetter N, Mefford HC, Smith RJH, Stephens K (Eds.), GeneReviews(®) [Internet]. Seattle (WA), University of Washington, Seattle, 1993-2016. 2004 [updated 2016].

2. Choi JH, Kim H, Yoo HW. Two cases of citrullinaemia presenting with stroke. J Inherit Metab Dis 2006; 29: 182-183.

3. Häberle J, Vilaseca MA, Meli C, et al. First manifestation of citrullinemia type I as differential diagnosis to postpartum psychosis in the puerperal period. Eur J Obstet Gynecol Reprod Biol 2010; 149: 228-229.
4. Faghfoury H, Baruteau J, de Baulny HO, Häberle J, Schulze A. Transient fulminant liver failure as an initial presentation in citrullinemia type I. Mol Genet Metab 2011; 102: 413-417.

5. Degirmencioglu H, Oncel MY, Yurttutan S, et al. Citrullinemia with an atypical presentation: persistent hiccups, Case report. Arch Argent Pediatr 2014; 112: 206-208.

6. Singh A, Kapoor S, Maheshwari N. Stroke as a rare manifestation of classical citrullinemia. Indian J Pediatr 2014; 81: 1249-1250. 